\title{
Metacontrast with internal contours in target and mask
}

\author{
DONNA ARAND and WILLIAM N. DEMBER \\ University of Cincinnati, Cincinnati, Ohio 45221
}

\begin{abstract}
Duration thresholds were obtained under nonmasking and masking conditions ior both solid black disks and disks broken into 16 black and white wedge-shaped segments; the targets were masked by either solid black rings or rings broken into 16 black and white segments. The results of previous research which investigated separately the effects of target and mask segmentation enabled the prediction that the least amount of masking would occur when the target was segmented, regardless of mask type. Similarly, the greatest amount of masking was expected when the target was a solid black disk. The data clearly disconfirmed those predictions and revealed instead the overriding importance of target-mask similarity. Implications of that outcome for the interpretation of previous research were noted.
\end{abstract}

The purpose of this experiment was to join two lines of research which had not previously been combined. Both were concerned with the role of configurational variables in metacontrast, following leads from Werner's basic target-mask pair, a black disk surrounded by a black ring. In general, we manipulated configurational variables by introducing internal contours into the target and the masking figure. Our methodology departs from Werner's in that we have replaced phenomenal report with a two-alternative, spatial forced-choice indicator response. We do this by following the target stimulus by a pair of masking rings, one of which is empty and the other of which encloses the target stimulus. The subject's task is to say on each trial in which one of the two rings the target has been presented.

In one line of research, we investigated the effect on metacontrast masking of deleting varying proportions of the masking ring and of the manner in which the remaining ring contour is distributed around the target disk. Of particular relevance to the present study is the finding that masking effectiveness generally increased with increases in the number of black and white segments comprising the masking stimulus, until a mask comprised of 16 black and white segments is as effective as a solid black ring (Arand \& Dember, 1974).

The second line of research involved internal contours in the target stimulus. Werner had concluded that internally contoured targets were less susceptible to masking than were homogeneous targets. We confirmed that conclusion in an experiment employing targets that were black on one side of the diagonal and white on the other and comparing their detectability under masking con-

Based on a Master's thesis by Donna Arand at the University of Cincinnati. Our thanks are extended to Joel Warm for his helpful comments and criticism. This research was reported at the annual meeting of the Psychonomic Society, November 6-8, 1975 , in Denver, Colorado. Reprints may be obtained from William N. Dember, Department of Psychology, University of Cincinnati, Cincinnati, Ohio 45221. ditions with that of a homogeneous black target (Sherrick \& Dember, 1970). A series of experiments then followed to find out whether a quantitative relation existed between number of internal contours (or number of black and white segments) and target maskability.

In the first of those experiments (Cox \& Dember, 1970), and then in all that followed, we included a nonmasking condition, in case targets with differing numbers of internal segments happened to be differentially detectable. As it turned out, increasing the number of target segments made the targets increasingly difficult to detect. Relative to those control data on detectability per se, the targets did, as we had expected, become harder to mask as the number of segments increased. For example, we found that targets with 16 black and white segments were both difficult to detect and difficult to mask (see Dember \& Stefl, 1972).

To summarize, our two lines of research allowed the following two conclusions: (1) A solid black ring and a ring broken into 16 black and white segments should be equally effective as masking stimuli; (2) a 16-segmented target disk should be considerably harder to mask than a solid black disk.

On the basis only of those two conclusions, as summarized above, we expected that the least amount of masking would be exhibited by a 16-segmented target in combination with either a 16-segmented mask or a solid mask, and that the greatest amount of masking would occur in the combinations comprised of a black target disk and either a black masking ring or a segmented ring. In short, we expected that masking would be determined only by the nature of the target stimulus.

\section{PROCEDURE}

Data were obtained from eight paid subjects who were naive about the purpose of the experiment, but trained in the psychophysical task. All had normal or corrected-to-normal vision. Each subject was tested in each condition of the experiment, a test consisting of measuring a duration threshold by a variant of the 
method of limits-the double staircase method (Dixon \& Massey, 1969). The duration in question is that of the target stimulus. The masking field, with or without a masking figure, had a constant duration of $105 \mathrm{msec}$; its onset coincided with the termination of the target stimulus.

The stimuli were presented in a Scientific Prototype threefield tachistoscope. All three fields were illuminated at a level of $20 \mathrm{fL}$, with one field serving as both an adapting and a fixation field. Viewing was monocular. The location of the target stimulus was varied randomly from trial to trial; it always appeared in the exact center of one of the two masking rings, or for the nonmasking condition, where that ring center would have been if a masking figure had been employed. Disk diameter was about $23.5 \mathrm{~min}$ of arc, and the ring pairs subtended an angle of about $1 \mathrm{deg}, 19 \mathrm{~min}$. Stimulus presentation was foveal. The subject's task was simply to say "left" or "right," guessing when necessary.

The basic design called for two types of target figure (solid black or segmented) and three masking conditions (a blank field, a pair of black rings, and a pair of segmented rings). We added one further variation to the segmented mask condition which the data subsequently proved to be unnecessary. That is, when the segmented target and the segmented mask occur in combination, the black and white segments of target and mask can either be perfectly aligned or else out of alignment. Not knowing whether alignment would matter, we prepared two segmented masks, one perfectly aligned with the segmented target and the other rotated so that the black and white segments of the masking figure were one segment out of phase with those in the segmented target. Both masking figures were employed in combination with the black disk as well. In effect, then, we ran a total of eight conditions-two targets by four masks.

Each subject was tested twice in each of the eight conditions; the two threshold measures were averaged for purposes of data analysis. The order in which those 16 threshold measurements were made varied randomly, with a different random order being used for each of the eight subjects.

\section{RESULTS AND DISCUSSION}

Mean duration threshold in milliseconds, averaged over eight subjects, is given in Table 1 for each of the eight conditions. Those data were reduced to the four values in Table 2 , which shows the mean increment in duration threshold attributable to the masking stimulus, collapsing over the two orientations of the segmented mask.

Analysis of variance of the data summarized in Table 2 yielded no significant main effects, but a significant Target by Mask interaction $(\mathrm{F}=10.94, \mathrm{df}=1, \mathrm{p}<.05)$. It is obvious that our original expectation was strikingly

Table 1

Mean Duration Threshold (Milliseconds) as a Function of Target and Mask Type

\begin{tabular}{llcccc}
\hline & \multicolumn{4}{c}{ Mask } \\
\cline { 2 - 5 } & None & Black & $\begin{array}{c}\text { Seg- } \\
\text { mented } \\
(1)\end{array}$ & $\begin{array}{c}\text { Seg- } \\
\text { mented } \\
(2)\end{array}$ \\
\hline \multirow{2}{*}{ Target } & Segmented & 17.43 & 26.11 & 34.08 & 32.09 \\
& Black & 10.38 & 26.01 & 23.65 & 23.65 \\
\hline
\end{tabular}

Note-The black and white segments of the Segmented 1 mask were aligned with corresponding segments of the segmented target; those of the Segmented 2 mask were one segment out of alignment.
Table 2

Increment to Duration Threshold (Milliseconds) Imposed by Masking Stimuli

\begin{tabular}{llrc}
\hline & & \multicolumn{2}{c}{ Mask } \\
& & Black & Segmented \\
\hline \multirow{2}{*}{ Target } & Segmented & 8.68 & 15.65 \\
& Black & 15.63 & 13.27 \\
\hline
\end{tabular}

disconfirmed. In particular, the amount of masking in the segmented target-segmented mask combination was much too high. What emerges clearly from these results is the overriding importance of target and mask similarity. The greatest amount of masking occurs in those combinations in which the target and mask have the same internal confirguration-that is, when both are solid or both are segmented.

That outcome, though unexpected, is consistent with the results of other experiments, for example by Parlee (1969) and by Uttal (1970), which showed increased masking with increased target-mask similarity in regard to such dimensions as the length or the shape of the target and mask figures. It also seems to fit the multiple channel model of masking recently proposed by Weisstein, Ozog, and Szoc (1975).

These results have also suggested that the data from our earlier studies on segmented targets may reveal little more than an effect of target-mask similarity. That is, the relative resistance to masking exhibited in our previous experiments by multisegmented targets may not imply anything special about internal contours per se, but may simply reflect the fact that we were using solid black rings as masks and comparing the maskability of solid and segmented targets. However, there is some consolation in the present data for our earlier view in the apparent lack of symmetry in extent of masking between the two dissimilar combinations. That is, when target and mask are dissimilar, the lesser amount of masking is associated with the combination containing the segmented target. That, at least, is supportive of our previous emphasis on the importance of internal contours. Thus, it may be that both target-mask similarity and internal target segments affect masking, but with similarity the much more potent factor.

\section{REFERENCES}

Arand, D., \& Dember, W. N. Masking effectiveness and number of segments in the masking ring. Bulletin of the Psychonomic Society, 1974, 3, 127-128.

Cox, S.. \& Dember, W. N. Backward masking of visual targets with internal contours. Psychonomic Science, 1970, 19. 255-256.

Dember, W. N., \& STEFL, M. Backward enhancement? Science, 1972, 175, 93-95.

Dixon, J. W., \& Massey, F. J. Introduction to statistical analysis. New York: McGraw-Hill, 1969.

PARLEe, M. Visual backward masking of a single line by a single line. Vision Research, 1969, 9, 199-205. 
Sherrick, M. F., \& Demger, W. N. Visual backward masking and the area-detection relation. Psychonomic Science, $1970,19,127-128$.

UtTal, W. R. On the physiological basis of masking with dotted visual noise. Perception \& Psychophysics, 1970 , 7. 321-327.

Weisstein, N., Ozog, G., \& Szoc, R. A comparison and elaboration of two models of metacontrast. Psychological Review, 1975, 82, 325-343.
Werner, H. Studies on contour: I. Qualitative analyses. American Journal of Psychology, 1935, 47, 40-64.

(Received for publication January 5, 1976.) 